\title{
The Complexity Analysis and Control of Time-Delay OEM Supply Chain considering R\&D Efforts and Marketing Level
}

\author{
Daoming Dai, ${ }^{1,2}$ Xuanyu Wu ${ }^{D},{ }^{1}$ and Fengshan $\mathrm{Si}^{1}$ \\ ${ }^{1}$ School of Management Science and Engineering, Anhui University of Finance and Economics, Bengbu 233030, China \\ ${ }^{2}$ School of Management, Hefei University of Technology, Hefei 230009, China \\ Correspondence should be addressed to Xuanyu Wu; 20142392@aufe.edu.cn
}

Received 18 June 2020; Revised 22 August 2020; Accepted 20 September 2020; Published 8 October 2020

Academic Editor: Ramachandran Raja

Copyright ( 2020 Daoming Dai et al. This is an open access article distributed under the Creative Commons Attribution License, which permits unrestricted use, distribution, and reproduction in any medium, provided the original work is properly cited.

In this paper, we investigate a supply chain consisting of an OEM (i.e., original equipment manufacturer) and a CM (contract manufacturer), in which the OEM decides design effort level and marketing level, and the CM makes decision on product manufacturing effort level. We establish a three-dimensional discrete dynamic model with time delay. Firstly, the sufficient conditions for Neimark-Sacker bifurcation are obtained by using different combinations of decision delay periods as bifurcation parameters, and the effect of the adjustment speeds of decision variables on the system stability and impact of time delay on the system stability are discussed, respectively. Secondly, we perform numerical simulation of this model from the perspective of entropy theory. Finally, we propose two methods to control chaos. Results show that when the time delay or the adjustment speed of decision variable exceed a certain threshold, the system will be led into a chaotic state and the entropy of the system will increase. For alleviating the negative effects of chaotic systems, we introduce control parameters to make efficient control on the chaos. At the critical point, the critical value of the adjustment speed of design effort level (or marketing level) of OEM increases as the adjustment speed of the manufacturing effort level of CM decreases, and vice versa.

\section{Introduction}

By adding Huawei Technologies Co. Ltd. and its affiliates to the Bureau's Entity List, the Bureau of Industry and Security (BIS) of the U.S. Department of Commerce has prohibited U.S companies from selling related technologies and products to Huawei, whose suppliers, such as Intel, Qualcomm, Xilinx, and Broadcom, will have to stop providing it with high-end chips in next moths. In order to reduce the potential disruption risk, Huawei has been implemented its "spare tire program" for more than a decade, in which its self-designed chips has partly been replaced with US highend chips. By now, Huawei has independently developed a series of chips, and the Kirin series are the most representative ones. Since high-end chip manufacturing is a technology intensive, capital intensive, and extremely complex industry, Huawei's self-designed high-end chip production has been outsourced to TSMC (Taiwan Semiconductor Manufacturing Company). Obviously, Huawei who is OEM focuses on R\&D of Kirin series and marketing of the Huawei mobile phones including the chips, and TSMC is CM who is responsible for manufacturing of the chips. Huawei has been committed to its core business and enhanced its core competitiveness. In the second quarter of 2020, the market share of Huawei's mobile phone rose to the first place in the world. Thus, it is of a substantial significance to investigate OEM supply chain with R\&D and marketing.

The existing literature studies the coordination of the OEM supply chain by the perspective of operation research. Van et al. [1] examine the impact of four variants of vendormanaged inventory on channel cost and cost allocation in OEM supply chain comprised of a supplier and an OEM. Li et al. [2] explore a direct-sale closed-loop supply chain consisting of an OEM, a remanufacturer, and two advertising agents and analyze the impacts of the parameters of design and advertising on equilibrium and the profits of the parties. Shen et al. [3] investigate how design outsourcing affects the supply chain and analyze channel performance 
under an OEM strategy versus an ODM (i.e., Original Design Manufacturer) strategy. Niu et al. [4] investigate fashion supply chain including a supplier, a contract manufacturer, and a fashion brand and discuss the fashion brand's profit performances under OEM strategy or ODM strategy. Kong and Li [5] examine the elasticity operation and promotion strategy for OEM supply chain and propose a deep learning mechanism to improve the flexibility of the channel. If the OEM supply chain is explored from entropy theory perspective, can more interesting conclusions be made?

In recent years, there have been the growing concerns on R\&D in the supply chains. Huang et al. [6] present a collaborative $\mathrm{R} \& \mathrm{D}$ model based on costumers' selection behavior and analyze the joint decisions on $\mathrm{R} \& \mathrm{D}$ and pricing in a supply chain. Stefano and Montes-Sancho [7] explore how the interaction between dyadic and network relationships can contribute to increase the success of environmental $\mathrm{R} \& \mathrm{D}$ cooperation. Yoo and Seo [8] propose six models on three-stage supply chain consisting of a R\&D firm, a manufacturer, and a retailer and examine the effect of supply chain structure and players' power dynamics on R\&D decision and market performances in a supply chain. Taleizadeh and Moshtagh [9] propose a consignment stock scheme for a closed-loop supply chain with imperfect manufacturing processes, lost sales, and quality dependent on return. Taleizadeh and Noori-daryan [10] propose pricing, manufacturing, and inventory policies for raw material in a three-level supply chain. Zhang et al. [11] present an implementation scheme of manufacturers considering remanufacturable design level under the multiperiod decision-making environment. $\mathrm{Ma}$ et al. [12] investigate a two-stage supply chain including two competitive manufacturers and one retailer and illustrate the optimal green manufacturing level and pricing.

Marketing is also an interesting aspect of supply chain research. Kort et al. [13] discuss pricing decisions in dualchannel supply chain in the presence of optional contingent products. Yaghin [14] investigates a production planning problem in dyadic supply chain with multiperiod, multiproduct, multisite, and multi-sales channels. Golgeci and Kuivalainen [15] discuss the role of absorptive capacity and marketing-supply chain management alignment and analyze the potential impact of social capital on supply chain resilience. Chernonog [16] investigates inventory and marketing policies in a supply chain with a perishable product.

Some researchers analyze the dynamic characteristics of the supply chain by using the entropy theory. Elsadany and Awad [17] focus on the difference between price and quantity competition in a mixed duopoly game and address the behavior of a duopolistic Bertrand competition market with environmental taxes. Ma et al. [18] examine a multichannel supply chain and discuss the complexity and bullwhip effect of each channel. Mondal [19] analyzes the stationary solutions of the proposed model and their stability conditions by using Routh-Hurwitz criteria. Yang et al. [20] propose the event trigger control protocol for fractional order nonlinear multiagent supply chain finance. Li et al.
[21] explore a low-carbon dual-channel supply chain and discuss the effects of different parameter values on the price stability and utility of the supply chain system. In addition, Saravanakumar et al. [22] discuss the results of dissipative control for kinds of switched neutral time-delayed systems and obtain the sufficient conditions for boundedness. Saravanakumar et al. [23] use Lyapunov technique and LIM approach to investigate the problems of robust dissipative control for kinds of discrete-time systems.

What are the dynamic characteristics of an OEM supply chain with R\&D and marketing from perspective of entropy theory? This will be an interesting and challenging subject. So far, the OEM supply chain has been applied by many multinational high-tech companies, and many scholars have an increasing interest in the OEM supply chain. Since most of decision makers are risk-averse and egocentric, it is more interesting to investigate the dynamic characteristics of an OEM supply chain with the time delay. In this paper, we investigate an OEM supply chain consisting of an OEM and a CM, in which OEM first decides the design effort level and marketing level, and then CM makes decision on the manufacturing effort level of the product. We discuss the effect of the time delay of three decision variables (i.e., design effort level $g_{m}$, marketing level $\eta$, and manufacturing effort level $g_{s}$ ) on the OEM supply chain. We obtain the conditions for the time delay to induce the bifurcation of the system through theoretical proofs and numerical simulation. In addition, we analyze the conditions of the system's perioddoubling bifurcation induced by the adjustment speed of the decision variables.

Compared with the existing classical research studies on coordination of OEM supply chains from operation management perspective, in which most of distributors and retailers are completely rational. The proposed method in this paper is used to analyze the chaotic behavior of OEM supply chain with time delay from entropy theory perspective, in which the distributor and the retailer are characteristic of bounded rationality.

The remainder of this paper is organized as follows. Description and assumption of the problem are presented in Section 2. The model is established in Section 3, and Section 4 discusses the dynamic characteristics of the problem. Section 5 addresses the dynamic characteristics of the problem by numerical simulation. Section 6 proposes two methods to control chaos. Section 7 concludes the paper with discussion of the possible future research.

\section{Description and Assumption of the Problem}

2.1. Model Construction and Assumptions. We investigate an OEM supply chain with R\&D efforts including design effort level $g_{m}$ of the OEM and manufacturing effort level $g_{s}$ of the $\mathrm{CM}$. The OEM and CM are responsible for the entire lifecycle of their products for consumers, from conception, through development and eventually to marketing. The OEM determines the design effort level $g_{m}$ and marketing level $\eta$ of the products, and manufacturing of the products is outsourced to the CM who decides the manufacturing effort level $g_{s}$. 
The main assumptions of this paper are made as follows:

(i) The CM and the OEM are bounded rational.

(ii) One-time investment is only taken into account in this paper. When the design effort level, marketing level, and manufacturing effort level are $g_{m}, \eta$, and $g_{s}$ respectively, the OEM and CM will have to incur the one-time investment cost $k_{s} g_{s}^{2} / 2, k_{n} \eta^{2} / 2$, and $k_{m} g_{m}^{2} / 2$, where $k_{s}, k_{n}, k_{m}$ are the cost coefficient [24].

(iii) R \& D effort includes design effort level $g_{m}$ of the OEM and manufacturing effort level $g_{s}$ of the CM.

(iv) The OEM and the CM are risk-averse, so they adopt the delayed decision policy.

2.2. Symbolic Description. The meanings of $D, \lambda, \alpha, \eta, g_{s}, g_{m}, p_{s}, p_{m}, k_{s}, k_{m}$, and $k_{n}$ are described concisely in Table 1 .

Based on the existing literature [25], the functional form of market demand can be written as follows:

$$
D=\alpha+\lambda(1+\eta)\left(g_{s}+g_{m}\right)
$$

where $\alpha$ expresses the market demand scale unaffected by other market factors, $\lambda$ describes the consumers' sensitivity to R\&D efforts, and OEM's marketing level is denoted by $\eta$. $g_{s}$ and $g_{m}$ represent manufacturing effort level of CM and design effort level of OEM, respectively.

\section{Multiperiod Decision-Making Game Model with Time Delay}

The profit functions of the OEM and the CM can be written as follows:

$$
\begin{aligned}
& \pi_{m}=p_{m}\left[\alpha+\lambda(1+\eta)\left(g_{s}+g_{m}\right)\right]-\frac{k_{m} g_{m}^{2}}{2}-\frac{k_{n} \eta^{2}}{2} \\
& \pi_{s}=p_{s}\left[\alpha+\lambda(1+\eta)\left(g_{s}+g_{m}\right)\right]-\frac{k_{s} g_{s}^{2}}{2} .
\end{aligned}
$$

As mentioned above, the decision variable of $\mathrm{CM}$ is manufacturing effort level $g_{s}$ and the decision variables of OEM are design effort level $g_{m}$ and marketing level $\eta$. The marginal profit functions can be written as follows:

$$
\left\{\begin{array}{l}
\frac{\partial \pi_{s}}{\partial g_{s}}=\lambda(1+\eta) p_{s}-k_{s} g_{s} \\
\frac{\partial \pi_{m}}{\partial g_{m}}=\lambda(1+\eta) p_{m}-k_{m} g_{m} \\
\frac{\partial \pi_{m}}{\partial \eta}=\lambda p_{m}\left(g_{s}+g_{m}\right)-k_{n} \eta
\end{array}\right.
$$

Due to the complexity of the market, the OEM or the CM cannot acquaint entirely the complete information of the
TABLE 1: The parameter description for the system.

\begin{tabular}{lc}
\hline$D$ & The total quantity of market demand \\
$\lambda$ & Consumers' sensitivity to R\&D efforts \\
$\alpha$ & Market demand scale unaffected by other market factors \\
$\eta$ & OEM's marketing level \\
$g_{s}$ & Manufacturing effort level of CM \\
$g_{m}$ & Design effort level of products of OEM \\
$p_{m}$ & OEM's profit margin \\
$p_{s}$ & CM's profit margin \\
$k_{s}$ & Coefficient of manufacturing effort level cost \\
$k_{m}$ & Coefficient of design effort cost \\
$k_{n}$ & Coefficient of marketing level cost \\
\hline
\end{tabular}

other decision maker and predict accurately the true information of the market. Therefore, the CM and the OEM are bounded rational. The decisions in the current period are dependent on the corresponding decisions in the last period and its marginal profit. The decisions in period $t+1$ under bounded rational hypothesis are the corresponding decisions in period $t$ plus the variations of decisions at period $t$ [26]:

$$
\left\{\begin{array}{l}
\eta(t+1)=\eta(t)+v_{1} \eta(t)\left[\lambda p_{m}\left(g_{s}(t)+g_{m}(t)\right)-k_{n} \eta(t)\right], \\
g_{s}(t+1)=g_{s}(t)+v_{2} g_{s}(t)\left[\lambda(1+\eta(t)) p_{s}-k_{s} g_{s}(t)\right], \\
g_{m}(t+1)=g_{m}(t)+v_{3} g_{m}(t)\left[\lambda(1+\eta(t)) p_{m}-k_{m} g_{m}(t)\right],
\end{array}\right.
$$

where $v_{1}, v_{2}$, and $v_{3}$ stand for the adjustment speed of marketing level $\eta$, manufacturing effort level $g_{s}$, and design effort level $g_{m}$, respectively. When the marginal profit of some decision variable is positive (or negative) in period $t$, the corresponding decision variables will increase (or decrease) in period $t+1$.

Because the OEM and the CM are risk-averse, so they adopt the delayed decision policy. Therefore, the three-dimensional discrete dynamic model with time delay can be proposed, which is referred to as model I:

$$
\left\{\begin{array}{l}
\eta(t+1)=\eta(t)+v_{1} \eta(t)\left[\lambda p_{m}\left(g_{s}(t-\tau)+g_{m}(t-\tau)\right)-k_{n} \eta(t)\right] \\
g_{s}(t+1)=g_{s}(t)+v_{2} g_{s}(t)\left[\lambda(1+\eta(t)) p_{s}-k_{s} g_{s}(t-\tau)\right] \\
g_{m}(t+1)=g_{m}(t)+v_{3} g_{m}(t)\left[\lambda(1+\eta(t)) p_{m}-k_{m} g_{m}(t-\tau)\right] .
\end{array}\right.
$$

\section{Existence and Local Stability of Neimark-Sacker Bifurcation}

4.1. Positive Equilibrium Points and Characteristic Equation of Model I. Based on repeated game theory, after the decision variables' continuous adjustment for some periods, model I can achieve an equilibrium state when the OEM (or the $\mathrm{CM}$ ) may not improve his profit by first changing his own decision. The equilibrium points of the model I are $E_{1} \sim E_{6}$ : 


$$
\begin{aligned}
& E_{1}(0,0,0), \\
& E_{2}\left(0, \frac{\lambda p_{s}}{k_{s}}, \frac{\lambda p_{m}}{k_{m}}\right), \\
& E_{3}(-1,0,0), \\
& E_{4}\left(0,0, \frac{\lambda p_{m}}{k_{m}}\right), \\
& E_{5}\left(0, \frac{\lambda p_{s}}{k_{s}}, 0\right), \\
& E_{6}\left(\frac{\lambda p_{m}}{k_{n}-\lambda p_{m}}, \frac{\lambda^{2} k_{n} p_{s}}{k_{s}\left(k_{n}-\lambda p_{m}\right)}, \frac{\lambda^{2} k_{n} p_{m}}{k_{m}\left(k_{n}-\lambda p_{m}\right)}\right) .
\end{aligned}
$$

The stability of each equilibrium point can be proved by solving its Jacobian matrix. Only if all the eigenvalues of its Jacobian matrix are less than 1, this equilibrium point is stable. Otherwise, it is unstable. The Jacobian matrix of model I can be written as follows:

$$
J=\left|\begin{array}{ccc}
T_{1} & -k_{s} v_{1} \eta & 0 \\
v_{2} g_{s} \lambda p_{m} & T_{2} & -k_{m} v_{2} g_{s} \\
v_{3} g_{s}\left(\lambda p_{m}-k_{n}\right) & 0 & T_{3}
\end{array}\right|,
$$

where

$$
\begin{aligned}
& T_{1}=1+v_{1}\left[\lambda(1+\eta) p_{s}-k_{s} g_{s}+\eta \lambda p_{s}\right] \\
& T_{2}=1+v_{2}\left[\lambda(1+\eta) p_{m}-k_{m} g_{m}\right] \\
& T_{3}=1+v_{3}\left[\lambda(1+\eta) p_{m}-k_{n} \eta\right] .
\end{aligned}
$$

First, judge the Jacobian matrix of equilibrium point $E_{1}(0,0,0)$ :

$$
J_{1}=\left|\begin{array}{ccc}
1+v_{1} \lambda p_{2} & 0 & 0 \\
0 & 1+v_{2} \lambda p_{m} & 0 \\
0 & 0 & 1+v_{3} \lambda p_{m}
\end{array}\right| .
$$

Obviously, all eigenvalues of $J_{1}$ are greater than 1 , so $E_{1}(0,0,0)$ is an unstable equilibrium point. Similarly, it can be proved that only $E_{6}\left(\eta^{*}, g_{s}^{*}, g_{m}^{*}\right)$ is a locally stable equilibrium point, and the others are unstable.

Let $\quad u_{1}(t)=\eta(t)-\eta^{*}, u_{2}(t)=g_{s}(t)-g_{s}^{*} \quad$ and $u_{3}(t)=g_{m}(t)-g_{m}^{*}$, then let $u_{1}(t)=\eta(t), u_{2}(t)=g_{s}(t)$, and $u_{3}(t)=g_{m}(t)$. For simplicity, $E_{6}\left(\eta^{*}, g_{s}^{*}, g_{m}^{*}\right)$ is transformed into the point $(0,0,0)$. First-order Taylor expansion of equation (5) at the equilibrium point $E_{6}$ is given:

$$
\left\{\begin{array}{l}
\eta(t+1)=a_{1} \eta(t)+a_{6}\left(g_{s}(t-\tau)+g_{m}(t-\tau)\right), \\
g_{s}(t+1)=a_{2} \eta(t)+a_{3} g_{s}(t)+a_{7} g_{s}(t-\tau), \\
g_{m}(t+1)=a_{4} \eta(t)+a_{5} g_{m}(t)+a_{8} g_{m}(t-\tau),
\end{array}\right.
$$

where

$$
\begin{aligned}
& a_{1}=1+v_{1}\left[\lambda\left(1+\eta^{*}\right) p_{s}-k_{s} g_{s}^{*}\right]+v_{1} \eta^{*} \lambda p_{s}, \\
& a_{2}=v_{2} g_{s}^{*} \lambda p_{m}, \\
& a_{3}=1+v_{2}\left[\lambda\left(1+\eta^{*}\right) p_{m}-k_{m} g_{m}^{*}\right], \\
& a_{4}=v_{3} g_{m}^{*} \lambda p_{m}, \\
& a_{5}=1+v_{3}\left[\lambda\left(1+\eta^{*}\right) p_{m}-k_{n} \eta^{*}\right], \\
& a_{6}=-v_{1} k_{s} \eta^{*}, \\
& a_{7}=-v_{2} k_{m} g_{s}^{*}, \\
& a_{8}=-v_{3} k_{m} g_{m}^{*} .
\end{aligned}
$$

Next, the characteristic determinant of model I can be written as follows:

$$
\left|\begin{array}{ccc}
\lambda-a_{1} & -a_{6} e^{-\lambda \tau} & -a_{6} e^{-\lambda \tau} \\
-a_{2} & \lambda-a_{3} & -a_{7} e^{-\lambda \tau} \\
-a_{4} & 0 & \lambda-a_{5}-a_{8} e^{-\lambda \tau}
\end{array}\right|
$$

And then, the characteristic polynomial of model I can be obtained:

$$
F(\lambda)=\lambda^{3}+\lambda^{2} B_{1}+\lambda B_{2}-B_{3}+\left[-a_{8} \lambda^{2}+\lambda B_{4}+B_{5}\right] e^{-\lambda \tau}+B_{6} e^{-2 \lambda \tau},
$$

where

$$
\begin{aligned}
& B_{1}=-a_{1}-a_{3}-a_{5}, \\
& B_{2}=a_{1} a_{3}+a_{1} a_{5}+a_{3} a_{5}, \\
& B_{3}=a_{1} a_{3} a_{5}, \\
& B_{4}=a_{1} a_{8}+a_{3} a_{8}-a_{4} a_{6}-a_{2} a_{6}, \\
& B_{5}=a_{2} a_{5} a_{6}-a_{1} a_{3} a_{8}, \\
& B_{6}=a_{2} a_{6} a_{8}-a_{4} a_{6} a_{7} .
\end{aligned}
$$

4.2. $\tau=0$, Sufficient Conditions for Local Stability at Equilibrium Point $E_{6}\left(\eta^{*}, g_{s}^{*}, g_{m}^{*}\right)$. When $\tau=0$, equation (13) can be simplified as follows:

$$
F(\lambda)=\lambda^{3}+\lambda^{2}\left(B_{1}-a_{8}\right)+\lambda\left(B_{2}+B_{4}\right)+B_{5}+B_{6}-B_{3} .
$$

Let $F(\lambda)=0$, according to Routh-Hurwitz criterion, if $\left(B_{1}-a_{8}\right)>0, \quad\left(B_{2}+B_{4}\right)>0$ and $\left(B_{1}-a_{8}\right) \quad\left(B_{2}+B_{4}\right)>$ $\left(B_{5}+B_{6}-B_{3}\right)$, the equilibrium point $E_{6}$ is locally asymptotically stable.

4.3. $\tau>0$, Sufficient Conditions for Local Stability at Equilibrium Point $E_{6}\left(\eta^{*}, g_{s}^{*}, g_{m}^{*}\right)$. Let $F(\lambda)=0$, and multiply both sides by $e^{\lambda \tau}$; then,

$$
-a_{8} \lambda^{2}+\lambda B_{4}+B_{5}\left(\lambda^{3}+\lambda^{2} B_{1}+\lambda B_{2}-B_{3}\right) e^{\lambda \tau}+B_{6} e^{-\lambda \tau}=0 .
$$


Assuming that $\lambda=i \omega(\omega>0)$ is the root of equation (16), then

$$
\left\{\begin{array}{l}
\Delta_{1} \sin (\omega \tau)+\Delta_{2} \cos (\omega \tau)=-a_{8} \omega^{2}-B_{5} \\
\Delta_{3} \sin (\omega \tau)-\Delta_{1} \cos (\omega \tau)=-\omega B_{4}
\end{array}\right.
$$

where

$$
\begin{aligned}
& \Delta_{1}=\omega^{3}-\omega B_{2}, \\
& \Delta_{2}=-\omega^{2} B_{1}+B_{6}-B_{3}, \\
& \Delta_{3}=-\omega^{2} B_{1}-B_{6}-B_{3} .
\end{aligned}
$$

In terms of equation (17), we have

$$
\omega^{12}+\omega^{10} C_{1}+\omega^{8} C_{2}+\omega^{6} C_{3}+\omega^{4} C_{4}+\omega^{2} C_{5}+C_{6}=0
$$

where

$$
\begin{aligned}
C_{1}= & 2\left(B_{1}^{2}-2 B_{2}\right)-a_{8}^{2}, \\
C_{2}= & \left(B_{1}^{2}-2 B_{2}\right)^{2}+2\left(2 B_{1} B_{3}+B_{2}^{2}\right)+2\left(a_{3}^{2} B_{2}+a_{8} B_{1} B_{4}-a_{8} B_{5}\right)-\left(a_{8} B_{1}+B_{4}\right)^{2}, \\
C_{3}= & 2\left(B_{3}^{2}-B_{6}^{2}\right)+2\left(B_{1}^{2}-2 B_{2}\right)\left(2 B_{1} B_{3}+B_{2}^{2}\right)+2\left(a_{8} B_{3} B_{4}-a_{3} B_{4} B_{6}+a_{3} B_{2} B_{5}\right) \\
& -\left(a_{8} B_{2}+B_{1} B_{4}-B_{5}\right)^{2}-2\left(a_{8} B_{1}+B_{4}\right)\left(B_{1} B_{5}+a_{8} B_{6}+a_{8} B_{3}-B_{2} B_{4}\right), \\
C_{4}= & \left(2 B_{1} B_{3}+B_{2}^{2}\right)^{2}+2\left(B_{1}^{2}-2 B_{2}\right)\left(B_{3}^{2}-B_{6}^{2}\right)-2\left(a_{8} B_{2}+B_{1} B_{4}-B_{5}\right)\left(B_{3} B_{4}-B_{4} B_{6}+B_{2} B_{5}\right) \\
& -2\left(a_{8} B_{1}+B_{4}\right)\left(B_{5} B_{6}+B_{3} B_{5}\right)-\left(B_{3} B_{5}+a_{8} B_{6}+a_{8} B_{3}-B_{2} B_{4}\right)^{2}, \\
C_{5}= & 2\left(2 B_{1} B_{3}+B_{2}^{2}\right)\left(B_{3}^{2}-B_{6}^{2}\right)-\left(B_{3} B_{4}-B_{4} B_{6}+B_{2} B_{5}\right)^{2} \\
& -2\left(B_{1} B_{5}+a_{8} B_{6}+a_{8} B_{3}-B_{2} B_{4}\right)\left(B_{5} B_{6}+B_{3} B_{5}\right), \\
C_{6}= & \left(B_{1}^{2}-2 B_{2}\right)^{2}-\left(B_{5} B_{6}+B_{3} B_{5}\right)^{2} .
\end{aligned}
$$

Define

$$
f(\omega)=\omega^{12}+\omega^{10} C_{1}+\omega^{8} C_{2}+\omega^{6} C_{3}+\omega^{4} C_{4}+\omega^{2} C_{5}+C_{6}=0
$$

We assume that $\left(H_{1}\right): f(\omega)$ has at least one positive real root. Without loss of generality, we assume that $f(\omega)$ has $n$ positive roots which are denoted by $f_{1}, f_{2}, \ldots, f_{n}, 0<n \leq 12$. From equation (17), we have

$$
\begin{aligned}
\tau_{k}^{(j)}= & \frac{1}{\omega_{k}} \arccos \left\{\frac{\Delta_{1} \omega B_{4}+\Delta_{3}\left(-a_{8} \omega^{2}-B_{5}\right)}{\Delta_{1}^{2}+\Delta_{2} \Delta_{3}}\right\} \\
& +\frac{2 j \pi}{\omega_{k}}, \quad k=1,2, \cdots, n ; j=0,1,2, \ldots
\end{aligned}
$$

Denote

$$
\begin{aligned}
\tau_{0} & =\min \left\{\tau_{k}^{(j)} \mid k=1,2, \ldots, n ; j=0,1, \ldots\right\} \\
& =\min \left\{\tau_{k}^{(0)} \mid k=1,2, \ldots, n\right\}=\tau_{k_{0}}^{(0)} .
\end{aligned}
$$

Differentiating both sides of equation (16) with regard to $\tau$ and substituting $\lambda=i \omega_{0}$ into the obtained expression, then we can obtain

$$
\operatorname{Re}\left[\frac{\mathrm{d} \lambda}{\mathrm{d} \tau}\right]_{\tau=\tau_{0}}^{-1}=\frac{R_{1} R_{2}+I_{1} I_{2}}{R_{1}^{2}+I_{1}^{2}}
$$

where

$$
\begin{aligned}
R_{1}= & \left(\omega_{0} B_{6}-\omega_{0}^{3} B_{1} B_{5}-\omega_{0} B_{3} B_{5}\right) \sin \left(\omega_{0} \tau_{0}\right) \\
& +\left(\omega_{0}^{2} B_{2} B_{5}-\omega_{0}^{4} B_{5}\right) \cos \left(\omega_{0} \tau_{0}\right), \\
I_{1}= & \left(-\omega_{0}^{4} B_{5}+\omega_{0}^{2} B_{2} B_{5}\right) \sin \left(\omega_{0} \tau_{0}\right) \\
& +\left(\omega_{0} B_{6}+\omega_{0}^{3} B_{1} B_{5}+\omega_{0} B_{3} B_{5}\right) \cos \left(\omega_{0} \tau_{0}\right), \\
R_{2}= & -2 \omega_{0} B_{1} B_{5} \sin \left(\omega_{0} \tau_{0}\right)+\left(B_{2} B_{5}-3 \omega_{0}^{2} B_{5}\right) \cos \left(\omega_{0} \tau_{0}\right) \\
& +B_{4}, \\
I_{2}= & \left(B_{2} B_{5}-3 \omega_{0}^{2} B_{5}\right) \sin \left(\omega_{0} \tau_{0}\right)+2 \omega_{0} B_{1} B_{5} \cos \left(\omega_{0} \tau_{0}\right) \\
& -2 a_{8} \omega_{0} .
\end{aligned}
$$

If condition $\left(H_{2}\right): R_{1} R_{2}+I_{1} I_{2} \neq 0$ holds, model I satisfies the condition of the occurrence for Neimark-Sacker bifurcation, so the following conclusions can be drawn.

Theorem 1. For model $I$, if conditions $\left(H_{1}\right),\left(H_{2}\right)$ hold, the equilibrium point $E_{6}\left(\eta^{*}, g_{s}^{*}, g_{m}^{*}\right)$ is asymptotically stable for $\tau \in\left[0, \tau_{0}\right)$; when $\tau=\tau_{0}$, model $I$ undergoes a Neimark-Sacker bifurcation at equilibrium point 
$E_{6}\left(\eta^{*}, g_{s}^{*}, g_{m}^{*}\right)$; model $I$ is unstable at equilibrium point $E_{6}\left(\eta^{*}, g_{s}^{*}, g_{m}^{*}\right)$ when $\tau>\tau_{0}$.

\section{Numerical Simulation}

In this section, we present some simulations to verify our conclusion. On the premise of satisfying the conditions listed in this paper, we take the parameter values as follows: $\lambda=0.248, p_{s}=1.9, p_{m}=1.8, k_{s}=0.37, k_{m}=0.2$, and $k_{n}=0.7$. We use the largest Lyapunov exponent and entropy to illustrate the features of the dynamic system. The principle of the largest Lyapunov exponent is that when exponent value is less than zero the system is stable, when exponent value is greater than zero, the system is unstable. According to the entropy theory, the more orderly the system, the lower its information entropy; conversely, the more chaotic the system, the higher its information entropy. Therefore, information entropy can also be said to be a measure of the degree of ordering of the system. When the entropy value of the system is zero, the system is so enough informative as to alleviate uncertainty, and it is in a stable state. By contrast, when the entropy value of system is more than zero, uncertainty of the system increases, and the system even goes into chaos. Thus, when the system is in a chaotic state, the decision makers have to collect more additional information to alleviate uncertainty.

5.1. Bifurcation Diagram Caused by Delay. By numerical simulation, $\tau_{0} \approx 0.286$. The bifurcation diagram of the system stability with respect to $\tau$ is shown in Figure 1.

As can be seen from Figure $1(\mathrm{a})$, when $\tau<\tau_{0}$, equilibrium point $E_{6}\left(\eta^{*}, g_{s}^{*}, g_{m}^{*}\right)$ is asymptotically stable; when $\tau>\tau_{0}$, model $\mathrm{I}$ is in a chaotic state and gradually produces Neimark-Sacker bifurcation from the stable state with the increase of $\tau$.

From Figure 1(a), it can be seen that when the time delay exceed a certain range, model I produces bifurcation or goes into chaos, which means that overdelayed decision-making will make the decision makers lose the best decision-making opportunity. Therefore, the OEM and the CM should make decisions in time to prevent the system from entering a chaos state. The entropy of model I with respect to $\tau$ is shown in Figure 1(b). When $\tau<0.286$, the entropy of model I is equal to 0 , and model $I$ is in a stable state at this time; when $\tau>0.286$, the entropy of model I continues increasing, and model $\mathrm{I}$ is in a chaotic state at this time. Comparing Figure 1(a) with Figure 1(b), it can be seen that the entropy of the system is increasing rapidly when bifurcation occurs and gets in chaos. Similarly, comparing Figure 1(a) with Figure 1(c), when bifurcation occurs and the system gets in chaos, the results of the largest Lyapunov exponent are consistent with that of bifurcation diagram, as shown in Figure 1(a).

\subsection{Bifurcation Diagram Caused by Adjustment Speed of} Decision. Ceteris paribus, when $\tau=0.01$, the influence of time delay on the stability of the system can be eliminated.
Then, we study the influence of adjustment speed on the stability by using 2D bifurcation diagram. First of all, when $v_{2}$ is fixed at $0.95, v_{3}$ is fixed at 0.85 , and $v_{1}$ changes from 0 to 0.5 , we discuss the effect of the adjustment speed $v_{1}$ of marketing level $\eta$ on the stability of model I. From Figure 2(a), if $v_{1}$ increases from 0 to 0.195 , model $\mathrm{I}$ is in a stable state, that is, the design effort level $g_{m}$, marketing level $\eta$, and manufacturing effort level $g_{s}$ stay at the equilibrium solution $E_{6}$; if $v_{1}=0.195$, the first bifurcation of the system occurs and the system turns into stable cycles of period 2, which means that the design effort level $g_{m}$, marketing level $\eta$, and manufacturing effort level $g_{s}$ have two possible values; with the further increase of $v_{1}$, the design effort level $g_{m}$, marketing level $\eta$, and manufacturing effort level $g_{s}$ become chaotic, that is, each decision variable has a lot of possible values.

Numerical simulations made by the largest Lyapunov exponent and entropy are shown in Figures 2(c) and 2(d). When $v_{1}=0.195$, the largest Lyapunov exponent reaches the first zero, and model I shows the bifurcation phenomenon; when the largest Lyapunov exponent is more than zero, model I goes into chaos. Similarly, when $v_{1}<0.195$, the entropy of model $\mathrm{I}$ is equal to 0 , and model $\mathrm{I}$ is in a stable state; when $v_{1}>0.195$, the entropy of model I continues to increase, period-doubling bifurcation occurs, and model I falls into chaos finally.

Compared with Figure 2(a), Figure 2(b) shows the change of the stability of model I with $v_{1}$ when $v_{2}$ reduces to 0.78 . Obviously, with the decrease of adjustment speed $v_{2}$ of the CM's manufacturing effort level $g_{s}$, the critical point of the stability of model I is shifted to the right, that is, as the adjustment speed $v_{2}$ of manufacturing effort level decreases, the critical value of adjustment speed $v_{1}$ of marketing level increases.

Let $v_{1}=0.5, v_{3}=0.52$, and $v_{2}$ varies from 0 to 0.5 , and we analyze the effect of the adjustment speed $v_{2}$ of manufacturing level $g_{s}$ on the stability of model I. (i) When $v_{2}$ increases from 0 to 0.15 , the design effort level $g_{m}$, marketing level $\eta$, and manufacturing effort level $g_{s}$ are fixed at the equilibrium point in Figure 3(a), the largest Lyapunov exponent is less than zero in Figure 3(c), the entropy is equal to zero in Figure 3(d), and model I is in a stable state in the three Figures above. (ii) When $v_{2}$ continues increasing to 0.15 , the first bifurcation occurs in Figure 3(a), and model I turns into stable cycles of period 2, which means that each decision variable has two possible values; the largest Lyapunov exponent is equal to zero in Figure 3(c), and the entropy of the system is more than zero in Figure 3(d), so the system becomes chaotic in Figures 3(c) and 3(d). (iii)With the increase of $v_{2}$, model I goes into chaos, which means that the design effort level $g_{m}$, the marketing level $\eta$, or the manufacturing effort level $g_{s}$ has a lot of possible values in Figures 3(a)-3(c).

Comparing Figure 3(a) with Figure 3(b), we find that, with decrease of the adjustment speed $v_{3}$ of the design effort level $g_{m}$, the critical point of the stability is shifted to the right, that is, with decrease of the adjustment speed $v_{3}$ of the design effort level $g_{m}$, the critical value of adjustment speed $v_{2}$ of the manufacturing effort level $g_{s}$ increases. 


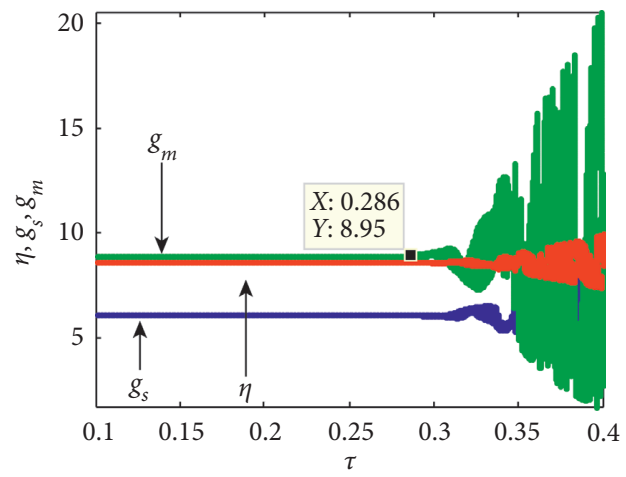

(a)

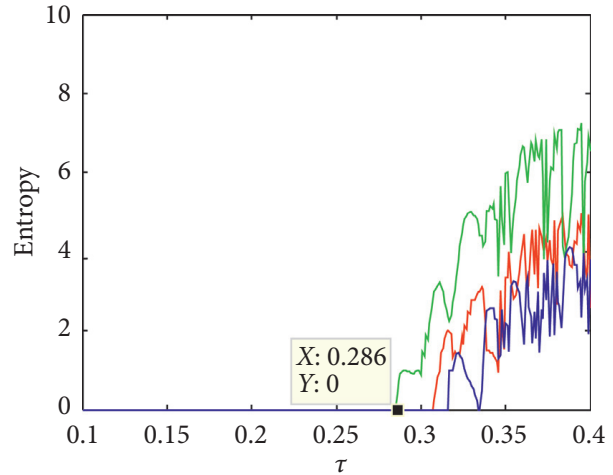

(b)

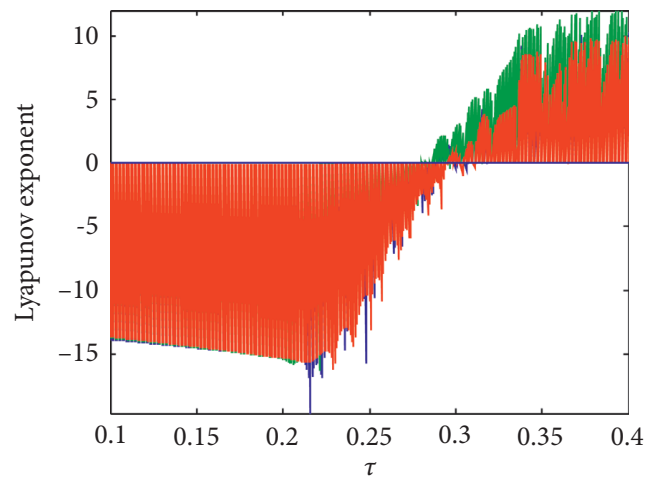

(c)

FIgUre 1: The impact of $\tau$ on the stability and complexity of model (I). (a) Bifurcation diagram of model I with. (b) Entropy diagram of Figure 1(a) respect to $\tau$. (c) Lyapunov exponents of Figure 1(a).

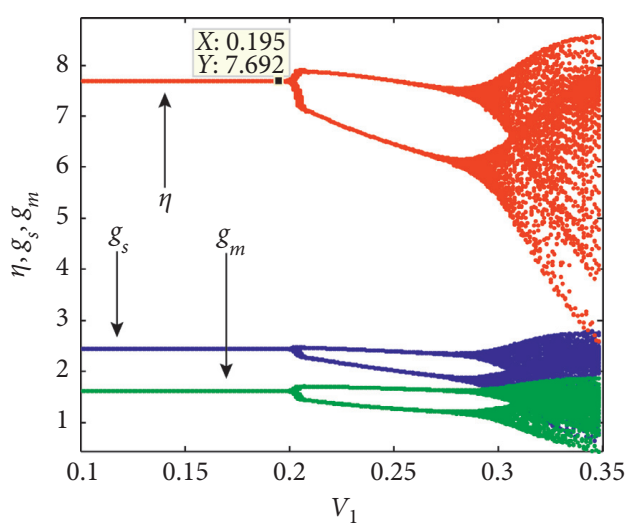

(a)

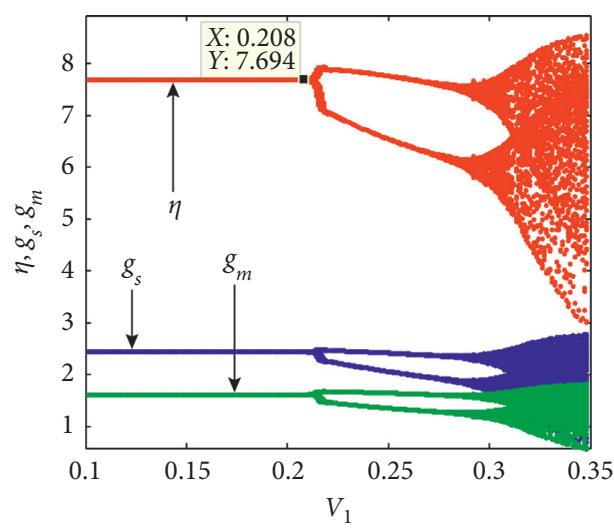

(b)

FIgURE 2: Continued. 


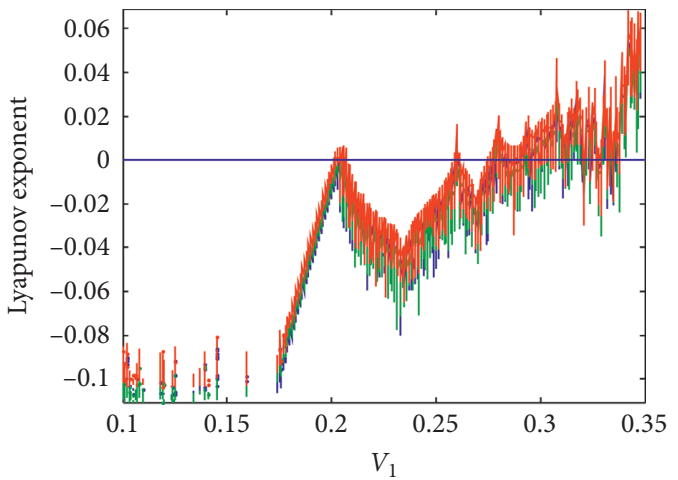

(c)

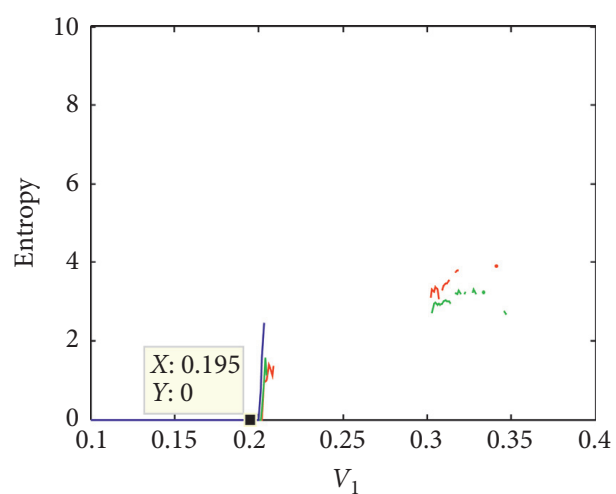

(d)

Figure 2: Dynamic evolution of decision variables with $v_{1}$. (a) Bifurcation diagram when $v_{2}=0.95$ and $v_{3}=0.85$. (b) Bifurcation diagram when $v_{2}=0.78$ and $v_{3}=0.85$. (c) Lyapunov exponents of Figure 2(a). (d) Entropy diagram of Figure 2(a).

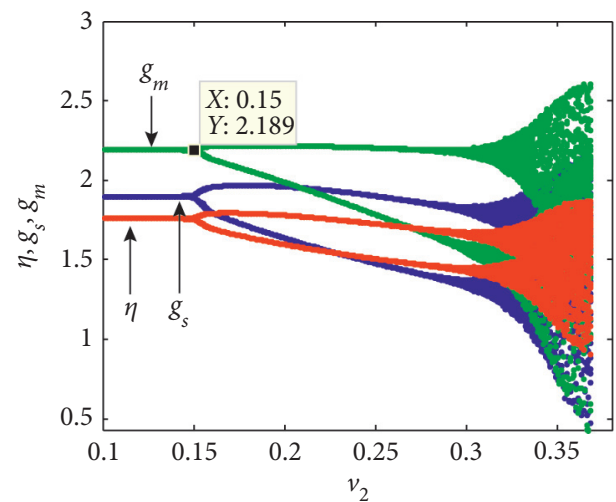

(a)

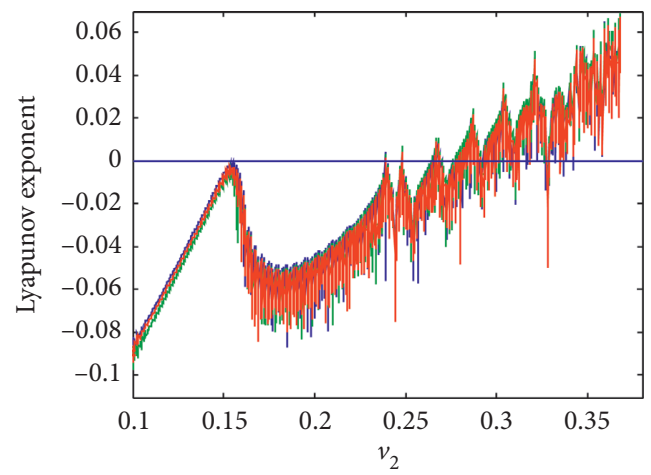

(c)

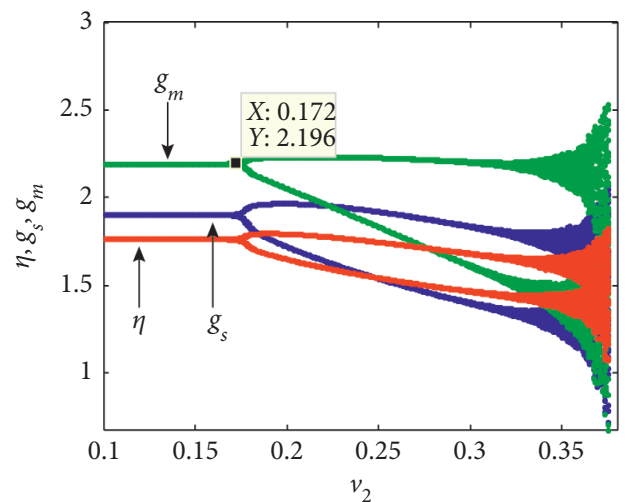

(b)

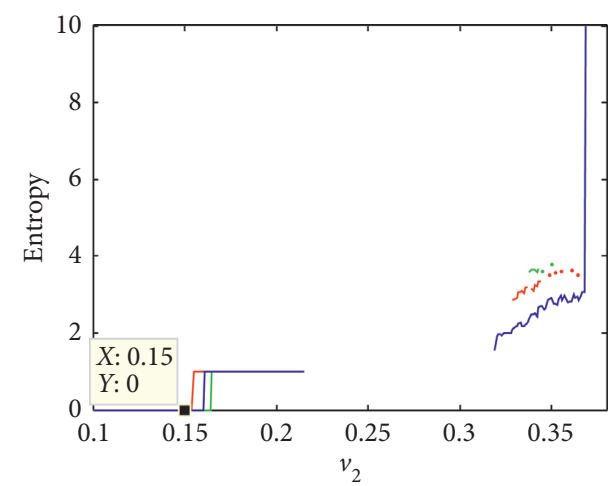

(d)

Figure 3: Dynamic evolution of decision variables with $v_{2}$. (a) Bifurcation diagram when $v_{1}=0.5$ and $v_{3}=0.52$. (b) Bifurcation diagram when $v_{1}=0.5$ and $v_{3}=0.33$. (c) Lyapunov exponents of Figure 3(a). (d) Entropy diagram of Figure 3(a).

Let $v_{1}=0.47, v_{2}=0.5$, and $v_{3}$ varies from 0 to 0.5 , we analyze the effect of the adjustment speed $v_{3}$ of design effort level $g_{m}$ on the stability of model I. (i)When $v_{3}$ increases from 0 to 0.136 , the design effort level $g_{m}$, marketing level $\eta$, and manufacturing level $g_{s}$ stay at the equilibrium point in Figure 4(a), and the largest Lyapunov exponent is less than zero in Figure 4(c), the entropy is equal to zero in
Figure $4(\mathrm{~d})$, and model $\mathrm{I}$ is in a stable state in the three Figures above. (ii) When $v_{3}$ continues increasing to 0.136 , the first bifurcation occurs in Figure 4(a), and model I turns into stable cycles of period 2, which means that each decision variable has two possible values; the largest Lyapunov exponent is equal to zero in Figure 4(c), and the entropy of the system is more than zero in Figure 4(d), so the system 


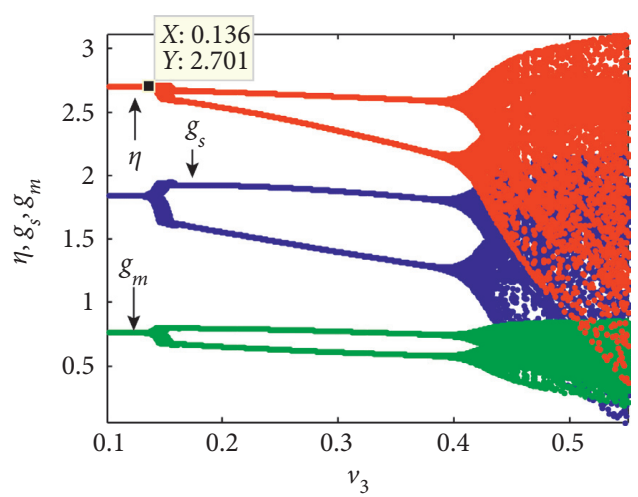

(a)

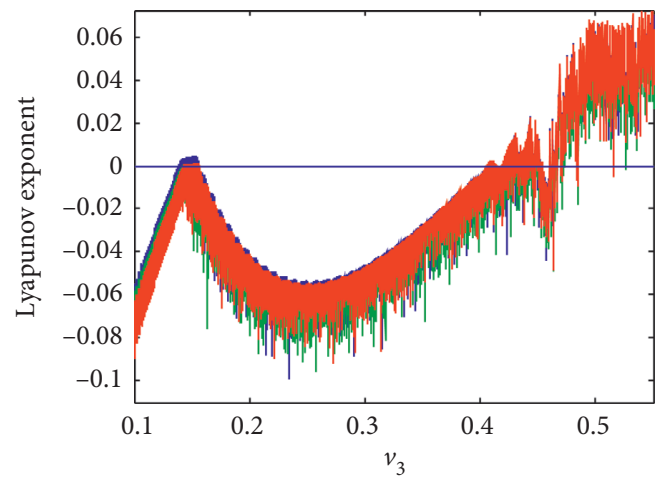

(c)

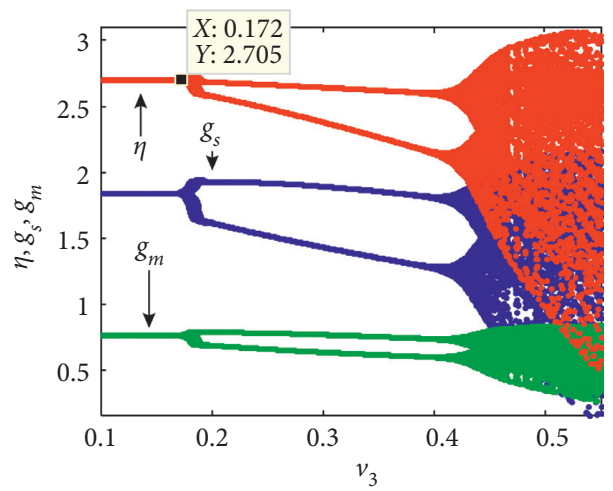

(b)

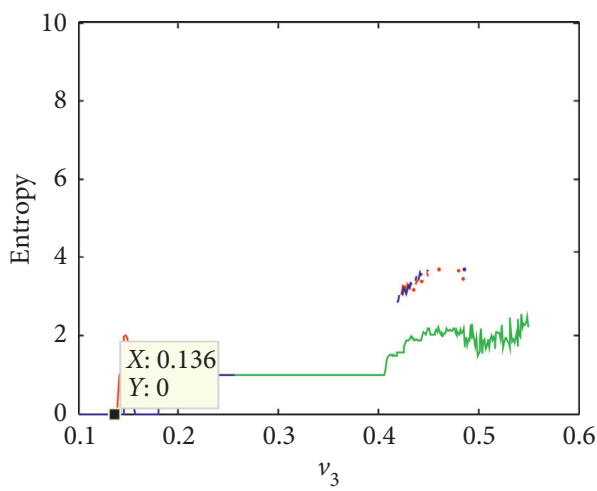

(d)

Figure 4: Dynamic evolution of decision variables with $v_{3}$. (a) Bifurcation diagram when $v_{1}=0.47$ and $v_{2}=0.5$. (b) Bifurcation diagram when $v_{1}=0.47$ and $v_{3}=0.45$. (c) Lyapunov exponents of Figure 4(a). (d) Entropy diagram of Figure 4(a).

becomes chaotic in Figures 4(c) and 4(d). (iii) With further increase of $v_{3}$, model I goes into chaos, which means that each of three decision variables has a lot of possible values in Figures 4(a)-4(c).

Comparing Figure 4(a) with Figure 4(b), a conclusion can be made that, with decrease of the adjustment speed $v_{2}$ of the manufacturing effort level $g_{s}$, the critical point of the stability is shifted to the right. That is, with decrease of the adjustment speed $v_{2}$ of the manufacturing effort level $g_{s}$, the critical value of adjustment speed $v_{3}$ of the design effort level $g_{m}$ increases.

Chaotic attractor is an important tool to characterize the chaotic state. When the system goes into a chaotic state, the structure of the chaotic attractor will be more complicated. Let $v_{1}=v_{2}=0.35$ and $v_{3}=0.5$. The system is in a chaotic state and Figure 5 illustrates the chaotic attractors.

From Figures 1-5, it can be seen that the faster the adjustment of the decision variables increases, the earlier the system gets into chaos. According to the entropy theory, when the system is chaotic, the entropy is high and the decision variables have a lot of possible values, which also causes that the decision maker make more effort to acquire useful information for alleviating uncertainty. Furthermore, the OEM and the CM should deliberate for decision time delay and the adjustment speed of the decision variables.

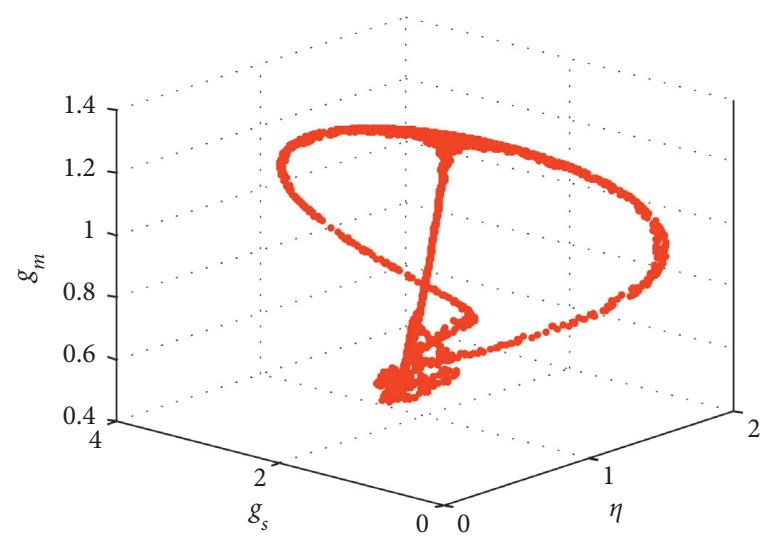

FIgURE 5: Chaotic attractor.

\section{Chaos Control}

From the previous discussion, both the time delay and the adjustment speed of the decision variables may cause the system to bifurcate, and even enter a chaotic state, which will always be harmful to make appropriate decision in a supply chain. Therefore, we try to control the chaos by the adjustment parameter control method and variable feedback control method, respectively. Let $\tau=0.5, v_{1}=v_{2}=0.35$, and 


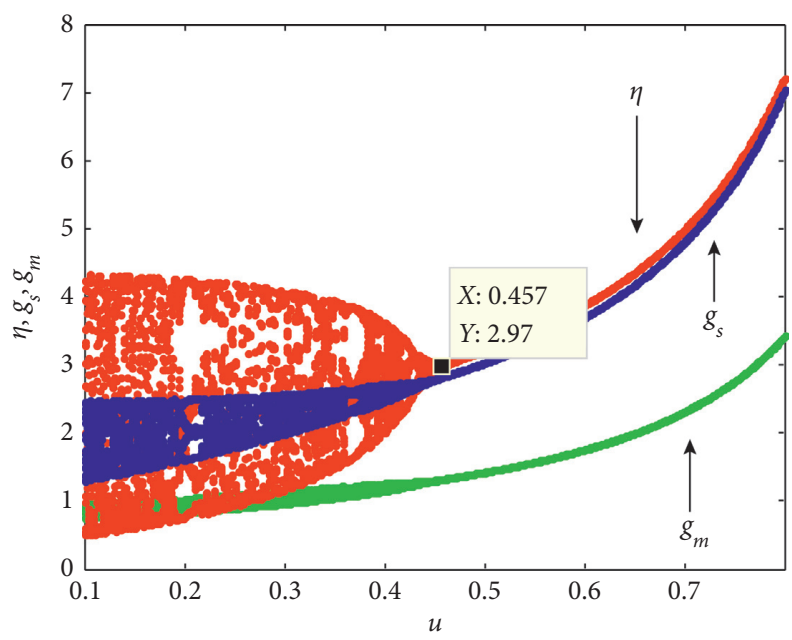

FIGURE 6: Systematic bifurcations with variation of adjustment parameter $u$.

$v_{3}=0.5$. As mentioned above, when other parameters keep fixed, model I is in a chaotic state.

6.1. Adjustment Parameter Control Method. In the adjustment parameter control method, the OEM and the CM cooperate with each other by jointly taking some measures to control the system chaos. Signing a contract is a common internal control measure.

The original model:

$$
\left\{\begin{array}{l}
\eta(t+1)=\eta(t)+v_{1} \eta(t)\left[\lambda p_{m}\left(g_{s}(t-\tau)+g_{m}(t-\tau)\right)-k_{n} \eta(t)\right] \\
g_{s}(t+1)=g_{s}(t)+v_{2} g_{s}(t)\left[\lambda(1+\eta(t)) p_{s}-k_{s} g_{s}(t-\tau)\right] \\
g_{m}(t+1)=g_{m}(t)+v_{3} g_{m}(t)\left[\lambda(1+\eta(t)) p_{m}-k_{m} g_{m}(t-\tau)\right]
\end{array}\right.
$$

After being controlled by parameter adjustment, the controlled model can be written as [27]

$$
\left\{\begin{array}{l}
\eta(t+1)=(1-u)\left\{\eta(t)+v_{1} \eta(t)\left[\lambda p_{m}\left(g_{s}(t-\tau)+g_{m}(t-\tau)\right)-k_{n} \eta(t)\right]\right\}+u \eta(t), \\
g_{s}(t+1)=(1-u)\left\{g_{s}(t)+v_{2} g_{s}(t)\left[\lambda(1+\eta(t)) p_{z}-k_{s} g_{s}(t-\tau)\right]\right\}+u g_{s}(t), \\
g_{m}(t+1)=(1-u)\left\{g_{m}(t)+v_{3} g_{m}(t)\left[\lambda(1+\eta(t)) p_{m}-k_{m} g_{m}(t-\tau)\right]\right\}+u g_{m}(t) .
\end{array}\right.
$$

Figure 6 illustrates that the chaos can be delayed or eliminated with a proper value of $u$. When $u=0$, the system is in the chaotic state. With the increasing of $u$, model I gets rid of chaos and goes into the stable state.

6.2. Variable Feedback Control Method. The main principle of the variable feedback control method is to use equation variables as control signals to eliminate chaos, which can be regarded as the regulation issued by the government for controlling the adjustment speeds of decision variables in the market. Compared with other control methods, this method has the advantage of only designing a simple controller. The controlled system can be expressed as follows [28]:

$$
\left\{\begin{array}{l}
\eta(t+1)=\eta(t)+v_{1} \eta(t)\left[\lambda p_{m}\left(g_{s}(t-\tau)+g_{m}(t-\tau)\right)-k_{n} \eta(t)\right]-u \eta(t) \\
g_{s}(t+1)=g_{s}(t)+v_{2} g_{s}(t)\left[\lambda(1+\eta(t)) p_{s}-k_{s} g_{s}(t-\tau)\right]-u g_{s}(t) \\
g_{m}(t+1)=g_{m}(t)+v_{3} g_{m}(t)\left[\lambda(1+\eta(t)) p_{m}-k_{m} g_{m}(t-\tau)\right]-u g_{m}(t)
\end{array}\right.
$$




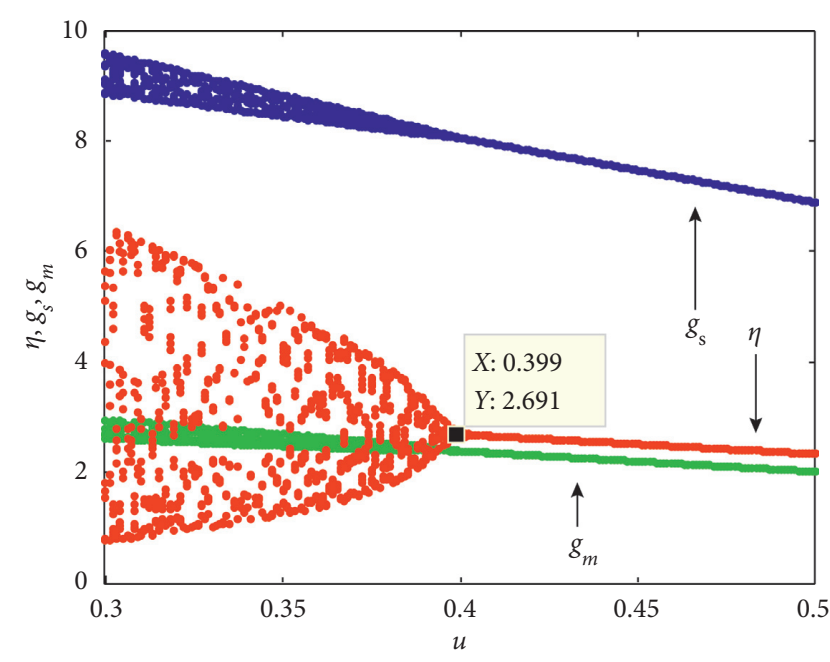

Figure 7: Systematic bifurcations with variation of adjustment parameter $u$.

The evolution process of model I with respect to $u$ is shown as Figure 7. When $u=0$, the system is in the chaotic state; when $u>0.457$, the system comes to a stable state and remains stable throughout, which indicates that the chaos of the system has been controlled effectively by some external intervention measures.

Figures 6 and 7 address the variation of model I under the adjustment parameter control and the variable feedback control, respectively. It is obvious that the control system in Figure 7 enters the stable state earlier than that in Figure 6. The control effect of the variable feedback control method is more efficient than that of the adjustment parameter control method because of the serious free riding in a supply chain. For controlling chaos may come with additional costs, the OEM and the CM have no incentive to cooperate with each other for controlling the system chaos. An unstable OEM supply chain can be efficiently transformed into a stable and orderly system through a relevant compulsory regulation issued by the government.

\section{Conclusion}

In this paper, we investigate a time delay supply chain including an OEM and a CM. The OEM decides product design effort level $g_{m}$ and marketing level $\eta$, and the CM makes decision on product manufacturing effort level $g_{s}$. Firstly, the mathematical description of the problem is formulated by three-dimensional discrete dynamic equations. Secondly, the positive equilibrium points and characteristic equation are discussed. The sufficient conditions for local stability at equilibrium point are analyzed. In addition, the effects of the adjustment speeds of decision variable on the decision variables are illustrated by using $2 \mathrm{D}$ bifurcation diagram, entropy, and chaotic attractor, respectively. Finally, the chaotic system can be transformed into the stable state by using the parameter control method and variable feedback control method, respectively. Several interesting conclusions are drawn as follows:
(1) The equilibrium point of the system is locally asymptotically stable when the value of the time delay $\tau$ is less than the critical value $\tau_{0}$; however, if the value of time delay $\tau$ is more than the critical value $\tau_{0}$, the system loses its stability and undergoes a Neimark-Sacker bifurcation. (2) If the adjustment speed of decision variable of the OEM or the CM increases to some threshold, the system will go into the chaotic state and the entropy of the system will increase. According to the entropy theory, when the entropy value of the system is low, the system is so enough informative as to alleviate uncertainty, and it is in a stable state. By contrast, when the entropy value of the system is high, uncertainty of the system increases and system even go into chaos. Thus, when the system is in a chaotic state, the decision makers have to collect more additional information to alleviate uncertainty. (3) At the critical point, as the adjustment speed of manufacturing effort level of CM decreases, the critical value of the adjustment speed of design effort level (or marketing level) of OEM increase, and vice versa. For example, ceteris paribus, the critical value of $v_{1}$ increases as $v_{2}$ decreases; the critical value of $v_{2}$ increases as $v_{3}$ decreases. (4) When the system is in a chaotic state, the internal control method and external control method can be generally used to eliminate chaos. For all parties in supply chain are so egoistic that they have difficulty in making a cooperation, the external control method can be more efficient than internal control method.

In the future research, we will take into account the impact of behavior factors on the system, such as altruistic preference and fairness concerns. In addition, it is an interesting subject to consider the fractional order equation as the form of demand function in an OEM supply chain.

\section{Data Availability}

The data used to support the findings of this study are available from the corresponding author upon request.

\section{Conflicts of Interest}

The authors declare no conflicts of interest.

\section{Acknowledgments}

The research was supported by the Key Project of Natural Science Research of Universities in Anhui Province (KJ2019A0662) and Graduate Research and Innovation Fund of Anhui University of Finance and Economics (ACYC2019205).

\section{References}

[1] P. L. M. Van Nyen, J. W. M. Bertrand, H. P. G. Van Ooijen, and N. J. Vandaele, "Supplier managed inventory in the OEM supply chain: the impact of relationship types on total costs and cost distribution," OR Spectrum, vol. 31, no. 1, pp. 167-194, 2009.

[2] Q. Li, H. Sun, H. Zhang, W. Li, and M. Ouyang, "Design investment and advertising decisions in direct-sales closed- 
loop supply chains," Journal of Cleaner Production, vol. 250, Article ID 119552, 2020.

[3] B. Shen, Q. Li, C. Dong, and V. Quan, "Design outsourcing in the fashion supply chain: OEM versus ODM," Journal of The Operational Research Society, vol. 67, no. 2, pp. 259-268, 2016.

[4] B. Niu, Y. Liu, L. Chen, and P. Ji, "Outsource to an OEM or an ODM? Profitability and sustainability analysis of a fashion supply chain," Journal of Systems Science and Systems Engineering, vol. 27, no. 4, pp. 399-416, 2018.

[5] F. H. Kong and J. Li, "Flexible operation and promotion strategy of OEM supply chain under the risk of supply interruption," China Management Science, vol. 26, no. 2, pp. 152-159, 2018.

[6] B. Huang, T. L. Saaty, and Y. Li, "Collaborative R\&D and pricing policy of supply chain under the selection behavior of heterogeneous customer," Mathematical Problems In Engineering, vol. 2019, 9 pages, 2019.

[7] M. C. De Stefano and M. J. Montes-Sancho, "Supply chain environmental R\&D cooperation and product performance: exploring the network dynamics of positional embeddedness," Journal of Purchasing And Supply Management, vol. 24, no. 4, pp. 288-303, 2018.

[8] S. H. Yoo and Y. W. Seo, "Effect of supply chain structure and power dynamics on R\&D and market performances," Journal of Business Economics and Management, vol. 18, no. 3, pp. 487-504, 2017.

[9] A. A. Taleizadeh and M. S. Moshtagh, "A consignment stock scheme for closed loop supply chain with imperfect manufacturing processes, lost sales, and quality dependent return: multi levels Structure," International Journal of Production Economics, vol. 217, pp. 298-316, 2019.

[10] A. A. Taleizadeh and M. Noori-daryan, "Pricing, manufacturing and inventory policies for raw material in a three-level supply chain," International Journal of Systems Science, vol. 47, no. 4, pp. 919-931, 2016.

[11] G. T. Zhang, X. Y. Qu, G. X. Dai, J. S. Hu, Y. B. Wang, and H. Sun, "Multi period closed-loop supply chain network equilibrium considering remanufacturing design level," China Management Science, vol. 26, no. 8, pp. 54-66, 2018.

[12] P. Ma, C. Zhang, X. Hong, and H. Xu, "Pricing decisions for substitutable products with green manufacturing in a competitive supply chain," Journal of Cleaner Production, vol. 183, pp. 618-640, 2018.

[13] P. M. Kort, S. Taboubi, and G. Zaccour, "Pricing decisions in marketing channels in the presence of optional contingent products," Central European Journal of Operations Research, vol. 28, no. 1, pp. 167-192, 2020.

[14] R. G. Yaghin, "Enhancing supply chain production-marketing planning with geometric multivariate demand function (a case study of textile industry)," Computers and Industrial Engineering, vol. 140, 2020.

[15] I. Gölgeci and O. Kuivalainen, "Does social capital matter for supply chain resilience? The role of absorptive capacity and marketing-supply chain management alignment," Industrial Marketing Management, vol. 84, pp. 63-74, 2020.

[16] T. Chernonog, "Inventory and marketing policy in a supply chain of a perishable product," International Journal of Production Economics, vol. 219, pp. 259-274, 2020.

[17] A. A. Elsadany and A. M. Awad, "Dynamics and chaos control of a duopolistic Bertrand competitions under environmental taxes," Annals of Operationas Research, vol. 274, no. 1-2, pp. 211-240, 2019.

[18] J. Ma, W. Lou, and Y. Tian, "Bullwhip effect and complexity analysis in a multi-channel supply chain considering price game with discount sensitivity," International Journal of Production Research, vol. 57, no. 17, pp. 5432-5452, 2019.

[19] S. Mondal, "A new supply chain model and its synchronization behaviour," Chaos, Solitons \& Fractals, vol. 123, pp. 140-148, 2019.

[20] S. Y. Yang, X. J. Chen, K. J. Leng, and W. Shi, "Research on complex dynamic behavior control of supply chain finance nonlinear system based on fractional differential operators," Chaos, vol. 29, no. 1, 2019.

[21] Q. X. Li, X. L. Chen, and Y. M. Huang, "The stability and complexity analysis of a low-carbon supply chain considering fairness concern behavior and sales service," International Journal of Environmental Research and Public Health, vol. 16, no. 15, Article ID 2711, 2019.

[22] T. Saravanakumar, V. J. Nirmala, R. Raja, J. Cao, and G. Lu, "Finite-time reliable dissipative control of neutral-type switched artificial neural networks with non-linear fault inputs and randomly occurring uncertainties," Asian Journal of Control, 2019.

[23] T. Saravanakumar, S. Marshal Anthoni, and Q. X. Zhu, "Resilient extended dissipative control for Markovian jump systems with partially known transition probabilities under actuator saturation," Journal of the Franklin Institute, vol. 357, no. 10, pp. 6197-6227, 2020.

[24] X. Chen, X. Wang, and M. Zhou, "Firms' green R\&D cooperation behaviour in a supply chain: technological spillover, power and coordination," International Journal of Production Economics, vol. 218, pp. 118-134, 2019.

[25] G. X. Lou, Y. H. Qiu, and H. Y. Xia, "Supply chain coordination of complex green products considering R\&D efforts and marketing investment," Industrial Engineering and Management in Chinese, http://kns.cnki.net/kcms/detail/31. 1738.T.20191104.1029.004.html, 2019.

[26] Q. X. Li, Y. H. Chen, Y. M. Huang, H. B. Gui, and S. Y. Liu, "The impacts of green innovation input and channel service in a dual-channel value chain," International Journal of Environmental Research and Public Health, vol. 16, no. 22, p. 4566, 2019.

[27] Q. X. Li, Y. H. Zhang, and Y. M. Huang, "The complexity analysis in dual-channel supply chain based on fairness concern and different business objectives," Complexity, 2018.

[28] R. Sakthivel, T. Saravanakumar, and M. Sathishkumar, "Nonfragile reliable control synthesis of the sugarcane borer," IET Systems Biology, vol. 11, no. 5, pp. 139-143, 2017. 